\title{
Clinical Efficacy of Sodium Deoxyribonucleate in the Treatment of Acute Respiratory Infections
}

\author{
Anna A. Zuikova, $\mathrm{PhD}, \mathrm{ScD}^{1}$; Olga N. Krasnorutskaya, $\mathrm{PhD}^{1}$; Julia A. Kotova, $\mathrm{PhD}^{1^{*}}$; \\ Daniel Yu. Bugrimov, $\mathrm{PhD}^{1}$; Olga V. Kashaeva, $\mathrm{PhD}^{2}$; Natalia M. Sizova ${ }^{3}$ \\ ${ }^{1}$ Voronezh State Medical University named after N.N. Burdenko, Russia \\ ${ }^{2}$ Moscow State University of Medicine and Dentistry named after A. I. Evdokimov, Russia \\ ${ }^{3}$ National Medical Research Center for Obstetrics, Gynecology and Perinatology named after \\ Academician V.I. Kulakov, Russia
}

\begin{abstract}
The aim of the study was to evaluate the effectiveness of sodium deoxyribonucleate (spray) in the treatment of patients with acute respiratory infections (ARI) in the outpatient setting.

Materials and Methods: We conducted a randomized controlled clinical trial. The study included 112 patients aged from 18 to 73 years with an established diagnosis of ARI and a duration of symptoms of the disease no more than 48 hours. The investigational drug - a $0.25 \%$ solution of sodium deoxyribonucleate (SD) for external and topical use (spray bottle, $10 \mathrm{ml}$ ). Group 1 included 54 patients who received SD: 2 intranasal doses into each nostril every 1-1.5 hours during the first days of the onset of the ARI symptoms; then - 2 doses into each nostril 3 times per day against the background of symptomatic therapy for 5 days. Symptomatic therapy included decongestants, antipyretics, mucolytics and antitussives (if necessary). Group 2 included 58 patients who received only symptomatic therapy. Medical examination was performed daily for 5 days.

Results: Including a $0.25 \%$ SD spray solution in the ARI treatment regimen effectively affects the dynamics of the ARI symptoms. SD in the treatment scheme from the first day allows eliminating the main ARI symptoms much faster and more efficiently than the standard treatment regimen; in particular, recovery is accelerated by 2-3 days. Complex therapy including SD causes a 3-fold increase in the level of SIgA in the nasal mucosa of patients on the fifth day of treatment, which indicates a pronounced immunomodulating effect of this intervention, a potential reduction in the risk of complications and recurrence of viral diseases.(International Journal of Biomedicine. 2019;9(1):52-56.)
\end{abstract}

Key Words: acute respiratory infections • secretory immunoglobulin A $\bullet$ squamous epithelium $\bullet$ symptomatic therapy

\section{Abbreviations}

ARI, acute respiratory infections; ADR, average degradation rate; FO, frequency of occurrence; sIgA, secretory immunoglobulin A; SD, sodium deoxyribonucleate; SE, squamous epithelium.

\section{Introduction}

Acute respiratory infections (ARI) are the leading cause of morbidity and mortality worldwide. ARI can occur in the upper and lower respiratory tracts and constitute a substantial

*Corresponding author: Prof. Anna A. Zuikova, PhD, ScD. Voronezh State Medical University named after N.N. Burdenko. Voronezh, Russia.E-mail: zuikova-terapia23@mail.ru disease burden in all age groups. ${ }^{(1,2)}$ They are the most common reason patients seek ambulatory care. ${ }^{(3-6)}$

In recent decades, there has been a tendency toward an increase in the prevalence of diseases of the upper respiratory tract, which cover an increasing number of the working population. Untreated, neglected and inadequately treated respiratory infections cause an increase in the incidence of, and contribute to the chronicity of, the diseases of the bronchopulmonary system, covering up to $7 \%$ of the total adult population of Russia. ${ }^{(2,7,8)}$ 
Diagnosis and treatment of ARI often cause certain difficulties for specialists. This circumstance is largely determined by the inability to establish the causative agent of the disease and the high cost of laboratory diagnosis, which, as a rule, is carried out in infectious hospitals and when an epidemically unfavorable situation occurs. ${ }^{(3)}$ ARI are a group of diseases caused by different microorganisms. A viral etiological agent is estimated to be present in up to $80 \%$ of cases. ${ }^{(9-11)}$ Influenza viruses are one of the main causative agents of ARI worldwide; however, many other respiratory viruses for which insufficient epidemiological information is available can also cause ARI. In the outpatient setting, it is extremely difficult to conduct a rapid diagnosis to identify the virus that caused the disease; therefore, the use of the term "acute respiratory viral infections" as a diagnosis is justified, since it indicates a nonbacterial etiology of the disease and makes it inappropriate to prescribe antibacterial medications. ${ }^{(2,7)}$ On average, adults suffer from ARI 2-3 times a year.

The polyetiologic nature of respiratory diseases does not allow one to use only specific vaccine preparations ${ }^{(8,12)}$ and dictates the need to use various nonspecific means ${ }^{(8,13,14)}$ that stimulate the natural resistance of the human body. In addition, immunomodulators are widely represented in the pharmaceutical market; however, it is extremely difficult to assess the real declared effectiveness. Many studies ${ }^{(3,5,12,15,16)}$ have shown that the maximum clinical effect of immunomodulating drugs in the complex treatment of ARI can be obtained with a combination of immunotropic action and reparative effects of drugs, which together form pronounced clinical efficacy. ${ }^{(13)}$

In light of the above, sodium deoxyribonucleate (SD) in the form of a "spray" is of certain interest for ARI therapy. The effects of this drug are due to the stimulation of the functional activity of T-helpers and T-killers, neutrophils and monocytes/ macrophages, ${ }^{(13,17)}$ and the enhancement of adaptive resources of mucosal immunity, which leads to the restoration of the epithelium lining the upper respiratory airways and potentiates local immunity. ${ }^{(18)}$

The aim of the study was to evaluate the effectiveness of SD (spray) in the treatment of ARI patients in the outpatient setting.

\section{Materials and Methods}

We conducted a randomized controlled clinical trial. The study included 112 patients aged from 18 to 73 years with an established diagnosis of ARI and a duration of symptoms of the disease no more than 48 hours.

Exclusion criteria: individual intolerance of the applied therapy; antiviral and immunomodulatory therapy; chronic diseases of the upper and lower respiratory tract; participation in any clinical trial during the previous month; violation of the procedure protocol. All participants provided the written informed consent.

The investigational drug - a $0.25 \%$ solution of SD for external and topical use (spray bottle, $10 \mathrm{ml}$ ).

The main group (Group 1) included 54 patients who received SD: 2 intranasal doses into each nostril every 1-1.5 hours during the first days of the onset of the ARI symptoms; then - 2 doses into each nostril 3 times per day against the background of symptomatic therapy for 5 days. Symptomatic therapy included decongestants, antipyretics (in cases of $\geq 38.5^{\circ} \mathrm{C}$ ), mucolytics and antitussives (if necessary). The comparison group (Group 2) included 58 patients who received only symptomatic therapy.

All patients underwent treatment in the therapeutic department at the Voronezh City Clinical Polyclinic No. 4. Examination of patients included collecting anamnesis, complete physical examination, complete blood count using the automated analyzer ADAMS-A1C HA-8160 (Arkray, Kyoto, Japan), urine analysis, direct immunofluorescence study of the nasal scrapings for viral antigens with a luminescent microscope of MIKMED-2 option 11 (Russia), determination of SIgA level on the IMMULITE 2000 XPi immunoassay system (Germany) and the surface layer condition of the epithelium using the Nikon ECLIPSE $\mathrm{Ni}-\mathrm{U}$ microscope (Germany).

To assess the state of cytological forms of the squamous epithelium of the nasal mucosa, the imprint smear technique was used. The manipulation was performed with a cotton swab dipped in sterile saline at the level of the inferior nasal concha. Rotational movements without pressure put smears on non-fat glass, dried, fixed and stained by RomanovskyGiemsa. Microscopy was performed under immersion with magnification $(7.0 \times 9.0)$. Two hundred cells were counted on a slide. In nasal smears, the number and cytoforms of squamous epithelium were determined with an assessment of the nature of the cell location, vacuolation of the cytoplasm and nuclei, dyschromia of the cytoplasm and nuclei, the shape and size of cells and nuclei, and the presence of multicore cells. To assess destructive processes according to the methodology proposed by L. Matveeva (1993), 5 classes of destruction $(0,1,2,3$, $4,5)$, cell destruction index (CDI), mean destruction index (MDI) and cell cytolysis index (CCI) were determined in the nasal mucosa.

Medical examination was performed daily for 5 days. The severity of the symptoms was assessed in points (0 complete absence, 1 - a mild severity, 2 - a medium severity, and 3 - a high severity). On the sixth day, at the end of the course of treatment, we registered complaints and conducted thorough physical and laboratory examinations. To determine the final score, the doctor counted the sum of points for each symptom separately. Thirty days after the start of treatment, the doctor interviewed patients by phone about well-being in the period since the end of the intake of the investigational drug. The safety and tolerability of the investigational drug was evaluated on the basis of an analysis of the frequency of occurrence (FO) of adverse events in all patients who received at least one dose of SD. The effectiveness of therapy was assessed according to the data obtained on the frequency of elimination of the pathogen, regression of clinical manifestations of ARI, the presence of complications, and repeated visits to the doctor caused by repeated episodes of respiratory infection. Symptom regression was considered to be 0 points, i.e. its complete absence.

Characteristics of the investigational drug

SD is an immunomodulatory agent, a regenerant, and a reparant. According to the literature, SD is an agonist for Toll- 
like receptor 9 (TLR-9, CD289). ${ }^{(13,17)}$ The immunomodulating effect of the drug is due to the interaction of the active substance (cytosine-guanine) with TLR9 on immunocompetent cells, which leads to the subsequent activation of a number of immune mechanisms. First, the stimulation of TLR in dendritic cells increases their ability to influence the differentiation of $\mathrm{T}$ helper cells in the direction of the formation of Type $2 \mathrm{~T}$ helper cells (Th2). Under the influence of Th2, B-lymphocytes differentiate into plasma cells secreting IgG2, IgG4, and IgM. Stimulated by TLR9, epithelial cells enhance the secretion of sIgA, which performs both the barrier function and the function of opsonin for interaction with the cellular element of the local immune response: macrophages and NK. Thus, stimulation of macrophage TLR9 with an increase in IFN $\gamma$ production leads to the activation of three levels of antiviral macrophage response.

Statistical analysis was performed using StatSoft Statistica v10.0 Baseline characteristics were summarized as frequencies and percentages for categorical variables and as mean \pm SEM for continuous variables. Student's unpaired and paired t-tests were used to compare two groups for data with normal distribution. Group comparisons with respect to categorical variables are performed using chi-square test. A probability value of $P<0.05$ was considered statistically significant.

\section{Results and Discussion}

During the initial examination, it was found that weakness and asthenia prevailed in patients of both groups. The febrile syndrome was most pronounced and manifested in 41 patients of Group 1 and in 46 patients of Group 2. FO for rhinorrhea, nasal congestion and oropharyngeal hyperemia (as components of the catarrhal syndrome) was $0.82,0.91$, and 0.65 in Group 1 and $0.78,0.88$, and 0.64 in Group 2, respectively. During the dynamic daily observation, it was found that FO for weakness and asthenia (symptoms of intoxication syndrome) was significantly lower $(P<0.05)$ in Group 1, starting from the third day of treatment, compared to Group 2: 0.39 and 0.44 against 0.86 and 0.86 , respectively. In Group 2, FO for these manifestations reached the values of Group 1 on day 5/6 of treatment. In the majority of patients of Group 1, the body temperature returned to a normal value on the third day of treatment; In Group 2, the value of $36.8^{\circ} \mathrm{C}$ was achieved only on the fifth day of treatment $(P<0.05)$. In Group 1, catarrhal phenomena in the oropharynx significantly decreased on the third day of treatment with FO of 0.22 $(P<0.05)$. A comparable $\mathrm{FO}$ of these phenomena in patients of Group 2 was achieved only by day 5/6 of therapy. In Group 1, in most cases, there was a clear positive trend in clinical symptoms: The nasal congestion almost completely disappeared by the end of the first day of treatment (FO was 0.12 on the second day of therapy); cough and symptoms of pharyngitis completely disappeared on the fourth day of treatment (FO was 0.03 on the third day of therapy). In Group 2 , the vector of changes in clinical symptoms was similar to the dynamics of patients in Group 1; however, the minimum OF for the symptoms was shifted by 2-3 days. Thus, FO for nasal congestion was 0.11 only by the sixth day of treatment, and the cough did not stop with FO of 0.21 by the seventh day. It was possible to achieve a regression of swelling of the tonsils and signs of pharyngitis in $20 \%$ of cases in Group 2 only by the sixth day of treatment. Differences in the regression of pharyngitis symptoms were statistically significant $(P<0.05)$. Compared to patients of Group 2, patients of Group 1 had a statistically higher incidence of regression for rhinorrhea symptoms by the third day of therapy - in $83 \%$ and $50 \%$ of cases, respectively $(P<0.05)$. In patients of both groups, the same percentage $(3 \%)$ of cases did not reveal the dynamics of rhinorrhea severity. After completion of treatment, the body temperature of all patients in Group 1 did not exceed normal values, while in Group 2, a subfebrile condition remained in one patient.

\section{$\underline{\text { IgA level assessment }}$}

Our analysis showed that the sIgA level in patients was lower the physiological norm by $65 \%(P<0.05)$ and amounted to $0.42 \pm 0.03 \mathrm{pg} / \mathrm{ml}$. In Group 2, the initial level of sIgA was reduced more than 3.5 times compared with the physiological norm. In Group 1, after therapy, the level of sIgA significantly increased almost 4 times and reached $1.64 \pm 0.02 \mathrm{pg} / \mathrm{ml}$ $(P<0.05)$. In Group 2, the sIgA level increased more than 2 times and reached $0.79 \pm 0.16 \mathrm{pg} / \mathrm{ml}$, but this growth did not reach the level of the physiological norm, making up only $70 \%$ of the normal level.

\section{Cytological characteristics of the nasal smears}

An analysis of the morphological picture of imprints from the nasal mucosa in patients from both groups prior to the start of therapy showed a significantly similar picture (Table 1): The proportion of free flat epithelial cells exceeded the physiological norm by 3 times; CDI was $54 \%$ higher than the physiological norm. A large number of pycnomorphic cells and cells with reduced tinctorial characteristics were present. This condition of the nasal mucosa fully reflected the picture of ARI with dominant catarrhal and intoxication syndromes. After treatment, in Group 1 there was a significant decrease in the content of free squamous epithelium to the values of the physiological norm, cytolysis and destruction of epithelial cells also decreased, and multicore cells were absent.

\section{Table 1.}

\section{Cytological characteristics of the nasal smears}

\begin{tabular}{|c|c|c|c|c|c|}
\hline \multirow[b]{2}{*}{ Variable } & \multirow[b]{2}{*}{$\begin{array}{c}\text { Physiological } \\
\text { norm }\end{array}$} & \multicolumn{2}{|c|}{ Group 1} & \multicolumn{2}{|c|}{ Group 2} \\
\hline & & $\begin{array}{c}\text { Before } \\
\text { treatment }\end{array}$ & $\begin{array}{c}\text { After } \\
\text { treatment }\end{array}$ & $\begin{array}{c}\text { Before } \\
\text { treatment }\end{array}$ & $\begin{array}{c}\text { After } \\
\text { treatment }\end{array}$ \\
\hline SE (\%) & 24.5 & $68.2 \pm 9.5^{*}$ & $26.4 \pm 2.1 *$ & $71.2 \pm 8.5^{*}$ & $51.3 \pm 4.5^{*}$ \\
\hline ADR & $0.53 \pm$ & $0.81 \pm 0.01$ & $0.52 \pm 0.01 *$ & $0.81 \pm 0.01 *$ & $0.69 \pm 0.05^{*}$ \\
\hline $\mathrm{CCI}$ & $0.04 \pm$ & $0.08 \pm 0.01$ & $0.04 \pm 0.01$ & $0.08 \pm 0.01$ & $0.06 \pm 0.02$ \\
\hline CDI & $0.48 \pm 0.08$ & $0.83 \pm 0.04$ & $0.41 \pm 0.03$ & $0.79 \pm 0.04 *$ & $0.64 \pm 0.03$ \\
\hline MDI & $0.41 \pm 0.01$ & $0.61 \pm 0.01$ & $0.39 \pm 0.02 *$ & $0.68 \pm 0.01 *$ & $0.64 \pm 0.01 *$ \\
\hline
\end{tabular}

$*_{-} P<0.05 ; A D R$ - average degradation rate; $S E$, squamous epithelium; CDI-cell destruction index; MDI- mean destruction index; CCI- cell cytolysis index. 
In Group 2, CDI and CCI decreased an insignificant amount as compared with the data before the start of therapy; there was a significant decrease (no more than $15 \%$ ) in the free epithelium, but it did not reach the level of the physiological norm; pycnomorphic flat epithelial cells were freely detected in the fields of view. Thus, it can be concluded that in Group 1 , by day 5 of therapy, the epithelium of the nasal mucosa had been morphologically restored and the possibility of local protection of the "entrance gate" of a viral infection was restored.

\section{The timing of the regression of ARI symptoms}

Analysis of the objective data from the physical examination led to the conclusion that the addition of SD to the symptomatic therapy in Group 1 contributed to a reliable regression of the pathological symptoms of ARI by the third to fourth day of therapy; a similar trend was observed in Group 2 by the sixth to seventh day. It should be noted that in Group 1 , on the second day of therapy, only 2 patients remained with moderately marked rhinorrhea compared with Group 2, where the same symptom was observed in 38 patients. By the second to third day of treatment in Group 1, nasal congestion was stopped in $95 \%$ of patients and in $32 \%$ of patients in Group 2. In Group 1, the swelling of the tonsils was stopped on the second day of treatment in $87 \%$ of patients, and on the third day was absent in all patients. In Group 2, this symptom was stopped in $39 \%$ of patients by the fourth day of therapy. By the end of therapy, catarrhal syndrome was more often absent in Group 1 patients. The cough was completely stopped in Group 1 by the fourth day of therapy and persisted until the sixth to seventh day in Group 2. Intoxication syndrome was completely stopped by the fourth day of therapy in Group 1 and continued up to the sixth to seventh day of observation in Group 2. Weakness and asthenia persisted in patients of Group 2 until the sixth day of therapy with FO of 0.36 and 0.15 , respectively. By the end of the observation, the need for symptomatic treatment had disappeared in $96 \%$ of patients in Group 1 and $86 \%$ of patients in Group 2; however, in Group 1 , the need for symptomatic treatment by the third day of therapy was significantly lower, by $60 \%$. Cases of undesirable reactions or side effects, which are not provided in the instructions for the medical use of SD (RU No. 002916 dated August 18, 2008), were not established during the study.

\section{Remote assessment of the effectiveness of therapy}

After the completion of therapy, the majority of patients in both groups (Group 1 - 46/85.2\% and Group 2 - 50/86.2\%) did not experience any symptoms of a past respiratory illness. Thirty days after the start of treatment, in order to detect repeated episodes of ARI and assess the safety of the treatment being performed, all patients were interviewed by phone. Repeated appeals regarding the symptoms of ARI within 30 days from the moment of discharge were recorded in $4 / 7.4 \%$ patients of Group 1 and in 16/27.6\% patients of Group 2. It should be noted that 4 patients in Group 2 developed acute bronchitis; therefore, the treatment of ARI was regarded as ineffective. Acute bronchitis was stopped for several days while patients were taking antibacterial drugs. Additional data on the manifestation of complications in patients of both groups were not identified.

\section{Discussion}

The main aim of this study was to analyze the effectiveness of a complex treatment of ARI with SD spray solution at a dose of $0.25 \mathrm{mg} / \mathrm{ml}$ with 2 sprays in each nostril 3 times a day for 5 days. The absence of any symptoms of ARI in patients of Group 1 against the inclusion of SD in the treatment regimen was already established by the third day of therapy, whereas in patients of Group 2, the disease symptoms (moderately pronounced rhinorrhea, cough, and nasal congestion) continued to be detected by day 6 . In patients of Group 1, the viral agent was eliminated by the time therapy was completed, which was not observed in patients of Group 2. The data of the multiparameter table of a medical examination show that after the first day of therapy in Group 1, the severity of ARI symptoms (rhinorrhea, cough, and nasal congestion) significantly decreased. The use of SD spray solution was not accompanied by any side effects. Statistically significant differences in the levels of sIgA were recorded in patients of 2 groups, which clearly demonstrated the effectiveness of SD as an immunomodulator and reparant of the epithelium of the nasal mucosa. Dynamic observation allowed us to establish a pattern of the disappearance of individual symptoms. Thus, by the third day of treatment in patients of Group 1, with the regression of catarrhal phenomena, nasal congestion and rhinorrhea occurred significantly more often; in addition, the swelling of the tonsils had a pronounced tendency to disappear more rapidly. Starting from the third day of therapy, patients of Group 1 were less likely to need additional medications. Complications of ARI developed in an insignificant percentage of patients in both groups of the study, and the identified conditions proceeded in moderate form and were stopped by standard antibacterial therapy. Practical results of treatment of ARI obtained by district therapists indicate that SD is able to form a stable immunity and induce the growth of local immune protection, restore the morphofunctional state of the mucous membranes, to inhibit the growth of pathogenic microflora, and increase the functional reserves of immunological protection. It has been clinically proven that during the first 12 hours after the administration of SD spray solution, such symptoms as rhinorrhea and tickling and dryness in the throat are relieved; mucous hyperemia and follicular edema of the posterior pharyngeal wall disappear by the end of the first day; the area of inflammatory lesions of the nasal mucosa is also significantly reduced.

\section{Conclusion}

As a result of the study, it was found that including a $0.25 \%$ SD spray solution in the ARI treatment regimen effectively affects the dynamics of the ARI symptoms:

1. SD in the treatment scheme from the first day allows eliminating the main ARI symptoms much faster and more efficiently than the standard treatment regimen; in particular, recovery is accelerated by 2-3 days.

2. Complex therapy including SD causes a 3-fold increase in the level of sIgA in the nasal mucosa of patients on the fifth day of treatment, which indicates a pronounced immunomodulating effect of this intervention, a potential reduction in the risk of complications and recurrence of viral diseases. 


\section{Competing Interests}

The authors declare that they have no competing interests.

\section{References}

1. Andreeva IV, Stetsyuk OU. [Respiratory tract infections: a new look at old problems]. Klinicheskaja mikrobiologija i antimikrobnaja himioterapijaerapy. 2009;11(2):143-51. [Article in Russian].

2. Lipatov MK. [The social significance of ARVI and the relevance of symptomatic treatment]. Russkii meditsinskii zhurnal. 2006;14(24):1569-74. [Article in Russian].

3. Zharkova NE. [Symptomatic treatment of ARVI: the future for combined drugs]. Russkii meditsinskii zhurnal. 2006;15(22):1636-9. [Article in Russian].

4. Morozova SV. [Treatment of acute upper respiratory tract infections]. Russkii meditsinskii zhurnal. 2005;13(26):174851. [Article in Russian].

5. Sinopalnikov AI, Belotserkovskaya YuG. [Influenza]. Lechachshii vrach.2007;(8):16-21. [Article in Russian].

6. Fernandes-Matano L, Monroy-Muñoz IE, AngelesMartínez J, Sarquiz-Martinez B, Palomec-Nava ID, PardavéAlejandre HD, et al. Prevalence of non-influenza respiratory viruses in acute respiratory infection cases in Mexico. PLoS One. 2017;12(5):e0176298. doi: 10.1371/journal.pone.0176298.

7. Gurov A.V. [Swelling of the mucous membrane of the upper respiratory tract. How to deal with it?] Russkii meditsinskii zhurnal. 2009;17(18):1-4. [Article in Russian].

8. Pchelintsev MV. [New clinical and pharmacological aspects of symptomatic treatment of ARVI and influenza]. Russkii meditsinskii zhurnal. 2009;17(14):924-8. [Article in Russian].

9. Bloom B, Cohen RA, Freeman G. Summary health statistics for U.S. children: National Health Interview Survey, 2008. Vital Health Stat 10. 2009;(244):1-81.

10. Mäkelä MJ, Puhakka T, Ruuskanen O, Leinonen M,
Saikku P, Kimpimäki M, Blomqvist S, Hyypiä T, Arstila P. Viruses and bacteria in the etiology of the common cold. J Clin Microbiol. 1998;36(2):539-42.

11. Shay DK, Holman RC, Newman RD, Liu LL, Stout JW, Anderson LJ. Bronchiolitis-associated hospitalizations among US children, 1980-1996. JAMA. 1999;282(15):1440-6.

12. Kryukov AI. [Symptomatic therapy in acute respiratory diseases]. Spravochnik poliklinicheskogo vracha. 2005;4(4):1236. [Article in Russian].

13. Filatov OYu, Kashaeva OV, Bugrimov DJu, Klimovich AA. [Morphophysiological principles of immunological effect of eukaryotic DNA]. Rossiiskii immunologicheskii zhurnal. 2013;7(4):385-90. [Article in Russian].

14. Gonzales P, Bartlett JG, Besser RE, Cooper RJ, Hickner JM, Hoffman JR, Sande MA. Principles of appropriate antibiotic use for treatment of acute respiratory tract infections in adults: background, specific aims, and methods.Ann Intern Med. 2001;134(6):479-86.

15. Gwaltney JM Jr, Druce HM. Efficacy of brompheniramine maleate for the treatment of rhinovirus colds. Clin Infect Dis. 1997;25(5):1188-94.

16. Prymula P, Siegrist CA, Chlibek R, Zemlickova H, Vackova M, Smetana J, et al. Effect of prophylactic paracetamol administration at time of vaccination on febrile reactions and antibody responses in children: two open-label, randomised controlled trials. Lancet. 2009;374(9698):133950. doi: 10.1016/S0140-6736(09)61208-3.

17. Vollmer J, Weeratna RD, Jurk M, Samulowitz U, McCluskie MJ, Payette P, et al. Oligodeoxynucleotides lacking CpG dinucleotides mediate Toll-like receptor 9 dependent $\mathrm{T}$ helper type 2 biased immune stimulation. Immunology. 2004;113(2):212-23.

18. Pikuza OI, Fayzullina RA, Suleymanova ZY, Zakirova AM, Serdinskaya IN. [Efficacy of Sodium deoxyribonucleate in community-acquired pneumonia in children of school age]. Kazan Medical Journal. 2017;98(5):736-742. [Article in Russian]. 